\title{
Curcumin-Loaded Chitosan/Gelatin Composite Sponge for Wound Healing Application
}

\author{
Van Cuong Nguyen, ${ }^{1}$ Van Boi Nguyen, ${ }^{1}$ and Ming-Fa Hsieh ${ }^{2}$ \\ ${ }^{1}$ Department of Chemical Engineering, Industrial University of Ho Chi Minh City, 12 Nguyen Van Bao Street, Go Vap, \\ Ho Chi Minh City 70000, Vietnam \\ ${ }^{2}$ Department of Biomedical Engineering, Center for Nanotechnology and Institute of Biomedical Technology, \\ Chung Yuan Christian University, 200 Chung Pei Road, Chungli, 32023 Taoyuan, Taiwan
}

Correspondence should be addressed to Van Cuong Nguyen; nvc@hui.edu.vn

Received 8 June 2013; Revised 4 July 2013; Accepted 4 July 2013

Academic Editor: Hai-Yin Yu

Copyright (C) 2013 Van Cuong Nguyen et al. This is an open access article distributed under the Creative Commons Attribution License, which permits unrestricted use, distribution, and reproduction in any medium, provided the original work is properly cited.

\begin{abstract}
Three composite sponges were made with $10 \%$ of curcumin and by using polymers, namely, chitosan and gelatin with various ratios. The chemical structure and morphology were evaluated by FTIR and SEM. These sponges were evaluated for water absorption capacity, antibacterial activity, in vitro drug release, and in vivo wound healing studies by excision wound model using rabbits. The in vivo study presented a greater wound closure in wounds treated with curcumin-composite sponge than those with composite sponge without curcumin and untreated group. These obtained results showed that combination of curcumin, chitosan and gelatin could improve the wound healing activity in comparison to chitosan, and gelatin without curcumin.
\end{abstract}

\section{Introduction}

Medicines derived from plants play an important role in the healthcare of many cultures, both ancient and modern. Curcumin (diferuloylmethane, a polyphenol) is an active agent of the perennial herb Curcuma longa (commonly known as turmeric). Curcumin is a naturally occurring polyphenolic phytoconstituent which presents anticancer, antioxidant, anti-inflammatory, hyperlipidemic, antibacterial, wound healing, and hepatoprotective activities $[1,2]$. The therapeutic efficacy of curcumin is limited due to its poor aqueous solubility and extensive first pass metabolism [35]. Topical formulation of curcumin (curcumin incorporated collagen matrix) was a feasible and productive approach to support dermal wound healing [1]. Therefore, development of novel curcumin formulation and delivery systems is required. The topical delivery of naturally occurring compounds, such as curcumin or catechins, is able to increase its solubility, stability, and pharmacological activities, resulting in improvement of therapeutic effects [6].
Additionally, chitosan, a polysaccharide biopolymer derived from naturally occurring chitin, displays unique polycationic, chelating, and film-forming properties due to the presence of active amino and hydroxyl functional groups. Chitosan also exhibits a number of interesting biological activities, including antimicrobial activity, induced disease resistance in plants, and diverse stimulating or inhibiting activities toward a number of human cell types $[7,8]$. Moreover, chitosan can be used to prevent or treat wound and burn infections due to its intrinsic antimicrobial properties and its ability to deliver extrinsic antimicrobial agents to wounds and burns [9]. Additionally, it can accelerate the functions of inflammatory cells, macrophages, and fibroblasts [10, 11]. Chitosan and its derivatives are also used to increase the stability of the drug in which the drugs are loaded in chitosan film or chitosan nanoparticles, resulting in enhancement of drug accumulation [12] and toxicity to cancer cells [13, 14]. Gelatin is also a natural polymer derived from collagen of animal skin and bones. It is biocompatible, hydrophilic, and biodegradable under normal physiological conditions. 
Gelatin is known to prevent fluid loss due to exudation, resulting in enhancement of its wound healing properties $[15,16]$. Thus, the present study focused on the preparation and evaluation of composite sponge made with two natural polymers: chitosan and gelatin at different proportions. Moreover, curcumin was loaded into the chitosan and gelatin sponge for wound healing application.

\section{Materials and Methods}

2.1. Materials. Chitosan is extracted from crab shells which was prepared from our lab. The degree of deacetylation of the chitosan was approximately $85 \%$. Curcumin was purchased from Vietnam Institute of Dietary Supplement (Hanoi, Vietnam) and acetic acid and gelatin were purchased from SigmaAldrich Chemical Co (USA). All chemicals used in this study were of reagent grade.

2.2. Preparation of Composite Sponge. Chitosan (1.0 g) was dissolved in $1 \%$ aqueous acetic acid $(100 \mathrm{~mL})$ to form a $1 \%$ chitosan solution (w/v) at room temperature. Gelatin (10\%) solution was prepared by dissolving gelatin powder $(10 \mathrm{~g})$ in $100 \mathrm{~mL}$ of deionized water at room temperature. The composite sponges were prepared by dissolving chitosan solution into the gelatin solution with different portions $(3: 1$, $1: 1$, and $1: 3$ ) of chitosan and gelatin, named as SP3, SP2 and $\mathrm{SP} 1$, respectively. The resulting solution was vigorously mixed under magnetic stirrer until the opaque aqueous solution was obtained. The solution was sonicated for $5 \mathrm{~min}$ for the removal of entrapped air bubbles. Then the air bubble-free solution was poured into separated petridishes and frozen at $-40^{\circ} \mathrm{C}$ for $24 \mathrm{~h}$ followed by lyophilization to obtain composite sponge. For curcumin encapsulated in composite sponge, $1 \mathrm{~mL}$ of curcumin solution $(0.5 \% \mathrm{w} / \mathrm{v}$ in absolute ethanol) was added to opaque aqueous solution. Then the solution was poured into separated petridishes, frozen at $-40^{\circ} \mathrm{C}$ for $24 \mathrm{~h}$, and followed by lyophilization to obtain curcumin composite sponge.

2.3. Characterization of Composite Sponge. The chemical structure and morphology of chitosan/gelatin composite sponges were evaluated using FT-IR (PerkinElmer) and SEM (JSM-5500).

2.4. Water Absorption Test. The water absorption capacity of the composite sponge was determined by incubating the composite sponge in phosphate-buffered saline (PBS) at $\mathrm{pH}$ 7.4 at $37^{\circ} \mathrm{C}$ for $2 \mathrm{~h}$. The wet weight of the swollen sponge was measured immediately after gently blotting with filter paper to remove absorbed water on surface, followed by lyophilization and reweighing. The water content of the sponge was calculated as follows:

$$
E_{\mathrm{ad}}=\left[\frac{W_{e}-W_{0}}{W_{0}}\right] \times 100,
$$

where $E_{\text {ad }}$ is the percentage water adsorption of chitosangelatin sponge at equilibrium. $W_{e}$ and $W_{0}$ represent the weight of the chitosan-gelatin sponge at equilibrium and initial weight of the chitosan-gelatin sponge, respectively.

2.5. Weight Loss of Prepared Sponges. The prepared sponges were immersed in PBS buffer solution at $37^{\circ} \mathrm{C}$. After $24 \mathrm{~h}$, the sponges were taken out, washed with deionized water, frozen, and lyophilized. The weights of sponges were measured after lyophilization. Weight loss of sponges was calculated through the following equation: $\% W=\left(W_{0}-W e\right) / W_{0} \times 100$, where $W_{0}$ and $W_{e}$ are the initial weight of sponge (g) and weight of sponge after lyophilization (g), respectively.

2.6. In Vitro Drug Release. The composite sponges $(3 \times 3 \mathrm{~cm})$ were dispersed in $20 \mathrm{~mL}$ phosphate buffer solution, $\mathrm{pH} 7.4$ at $37^{\circ} \mathrm{C}$. All the supernatants were pipetted out periodically and replaced with equivalent volume of fresh phosphate buffer solution. The concentration of released drug was then determined by UV-Vis spectrometer at $420 \mathrm{~nm}$ using a standard curve of curcumin in ethanol. The percentage of curcumin released was determined as curcumin release $(\%)=$ curcumin released at time $t$ /total curcumin loaded in sponge $\times 100$.

2.7. Evaluation of Antimicrobial Activity. The measurements of the antimicrobial activities of individual chitosan and composite sponge were conducted by agar diffusion method using Pseudomonas aeruginosa. The zone of inhibition was determined by placing a definite size of sponge in inoculated solidified nutrient agar medium in a petriplate. Petriplates were incubated for $24 \mathrm{~h}$ at $37^{\circ} \mathrm{C}$. This was done in triplicate with each film for each organism, and an average diameter of zone of inhibition was noted.

2.8. Cytotoxicity. The cytotoxicity of curcumin sponge was conducted on mouse fibroblast cells (L929) indirect MTT method [17]. The cells were seeded into 96-well microplates at a density of $10^{3}$ cells/well at $37^{\circ} \mathrm{C}$ under humidified atmosphere containing $5 \% \mathrm{CO}_{2}$. Triplicates of sterilized sample were taken and then incubated in serum containing media for $24 \mathrm{~h}$ at $37^{\circ} \mathrm{C} .100 \mu \mathrm{L}$ of the media from each sample was taken and transferred into each well then incubated for another $24 \mathrm{~h}$. After that, MTT solution was added to each well followed by 4 hours of incubation at $37^{\circ} \mathrm{C}$. Subsequently, the medium was removed and violet crystals formed were solubilized with DMSO. After shaking slowly twice for 5 seconds, the absorbance of each well was determined using Eliza reader at $570 \mathrm{~nm}$. The cell viability was expressed as a percentage of the control.

2.9. Evaluation of Wound Healing Activity. The wound healing activity was evaluated by excision wound model in albino rabbits ( 1.7 to $2.0 \mathrm{~kg}$ ). All in vivo experiments were carried out in accordance with the ethical guidelines for Animal Care and Use Committee of Medical and Pharmaceutical University (Ho Chi Minh City). The animals were anesthetized by using lidocaine $25 \%$ prior to the test. The dorsal hair of the rabbit was removed. Full thickness wound of $2.5 \times 2.5 \mathrm{~cm}^{2}$ was excised from the back of the rabbit. The wound was covered 
with an equal size of drug-loaded sponge or blank sponge. The cotton gauge was used for control. The sponge was removed and replaced by fresh sponge every three days. The wound closure was observed at different periods of treatment using a digital camera. Wound area was calculated on the 0th, 3rd, 12nd, 15th and 21st days. On day 21st, histological examinations of skin wound tissue were carried out. The skin wound tissue of animal was excised, fixed with $10 \%$ formalin, and stained with hematoxylin-eosin ( $\mathrm{H} \& \mathrm{E})$ reagent for histological observations.

\section{Results and Discussion}

The composite sponges were prepared in different ratios of chitosan and gelatin $(3: 1,1: 1$, and $1: 3)$ (Figure 1). The results indicated that increasing of gelatin concentration leads to increase of folding endurance. The composite sponge SP1 showed the highest folding endurance among all composite sponges (data not shown). To examine the chemical structures of various sponges prepared under different feeding ratios, the prepared sponges were examined by FTIR spectra. The $-\mathrm{CH}$ - stretching vibration of the chitosan and gelatin is at $2936-2969 \mathrm{~cm}^{-1}$, while peaks of chitosan at 1650 and $1560 \mathrm{~cm}^{-1}$ correspond to the amide carbonyl group (amide I) and the bending frequency of the amide $\mathrm{N}-\mathrm{H}$ group (amide II), respectively (Figure 2). The FT-IR of composite sponge exhibited a mixture of characteristic absorptions because of amine groups of chitosan and the carboxylic acid groups of gelatin. The peaks of $\mathrm{C}=\mathrm{O}$ of amide I for chitosan at $1650 \mathrm{~cm}^{-1}$ was shifted to $1669 \mathrm{~cm}^{-1}$ in composite sponges. The intensity of $\mathrm{C}=\mathrm{O}$ peaks increased with the decline ratio of gelatin in composite sponge from $1: 3$ to $3: 1$ (chitosan/gelatin). These results can be attributed to the interaction of $-\mathrm{NH}^{3+}$ of chitosan and the $\mathrm{COO}^{-}$of gelatin in three composites $[18,19]$.

Figure 3 shows the cross-section morphology of chitosan/gelatin composite sponges. According to the figure, the sponges appeared as a very porous structure. Additionally, the roughness of chitosan/gelatin sponge surface was decreased with increasing of gelatin content (Figures 3(a)-3(c)). It was found that the large pores were observed in cross-section morphology of chitosan/gelatin sponges (Figures 3(d)-3(f)). The pore sizes were $43.06,35.6$, and $29.9 \mu \mathrm{m}$ for SP3, SP2, and SP1, respectively. The sponges with low gelatin level represent regular pore size. On the other hand, the irregular pore size was observed in cross-section morphology of sponge with high gelatin content. The irregular pore size appearing in the sponge with high gelatin level may be deduced from the incompatibility between chitosan and gelatin molecules.

Water absorption capacity is an important parameter for biological applications and wound healing. It presents the capacity of sponge to absorb wound exudates. As the gelatin contents of prepared sponges were increased, the water adsorption capacities were significantly decreased. This is because lower water content led to smaller pore size during the preparation of the sponges. As a result, the water uptake was relatively lower. The water uptake capacity was $227 \%$, $351 \%$, and $2352 \%$ for ratio of chitosan/gelatin $1: 3,1: 1$, and

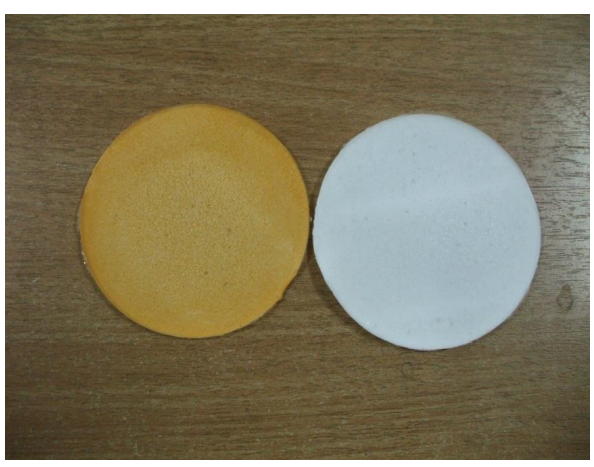

(a)

(b)

FIGURE 1: Prepared composite sponge with ratio of chitosan and gelatin $(3: 1)$ (a) curcumin-sponge and (b) blank sponge.

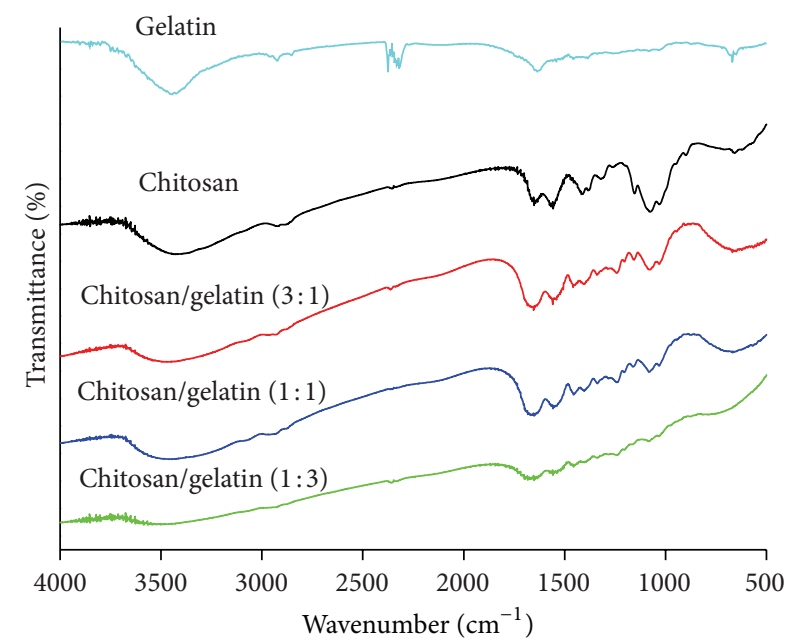

FIGURE 2: FT-IR spectra of prepared composite sponge with various ratios of chitosan and gelatin.

$3: 1$, respectively. The high water content in prepared sponges was explained by the hydrophilic and swelling properties of gelatin [20]. The SP3 presented maximum water absorption capacity among all prepared sponges; the sponge can retain water in porous pore surrounding by cell walls. Additionally, it decreased about 10-fold for SP1 and SP2 which may attribute to the swelling and dissolving of gelatin that could decrease the pore size leading to less water absorption and therefore could not retain much water within their network structure. Furthermore, the water absorption capacity of drug-loaded sponge was not significantly different from blank sponge. The similar results were also reported in previous study [18]. These results were further confirmed by weight loss of sponges. Table 1 presents that the weight losses were 81.5, 66.5 and $16 \%$ for SP1, SP2, and SP3, respectively. The weight loss is reasonable as more gelatin can resist the dissolution in water. The water uptake may be related to the pore. The more gelatin, the smaller pores, and pore volume were observed.

The in vitro release profile of curcumin-sponge was conducted in phosphate buffer solution. The average percent release of curcumin was approximately $91 \%, 55 \%$, and $37 \%$ for 


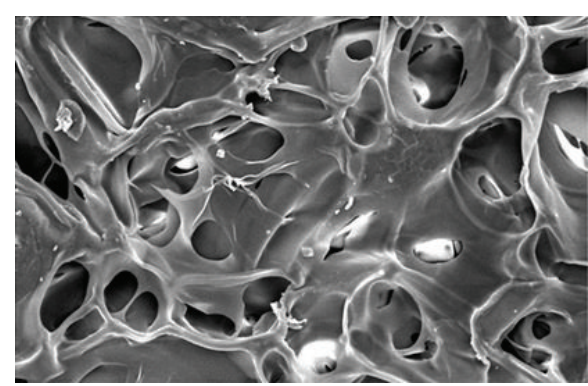

(a)

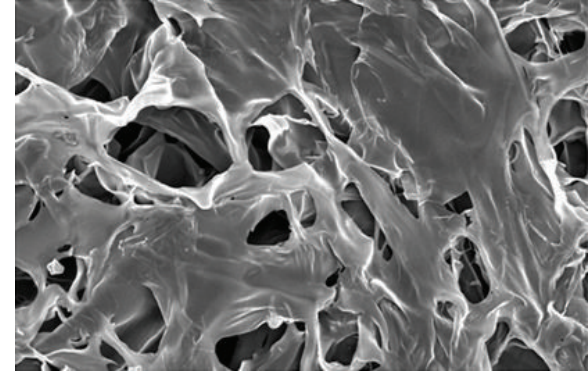

(b)

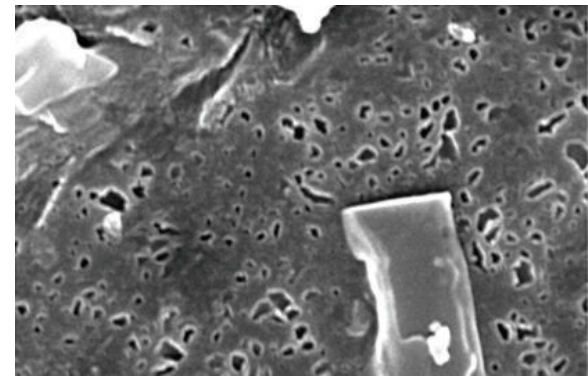

(c)

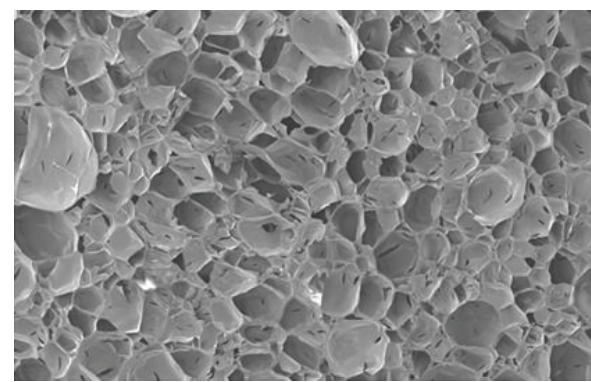

(d)

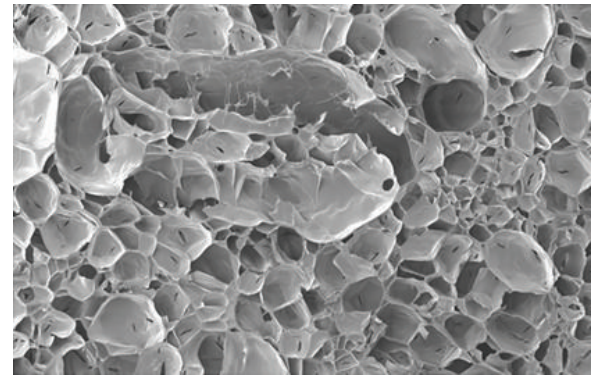

(e)

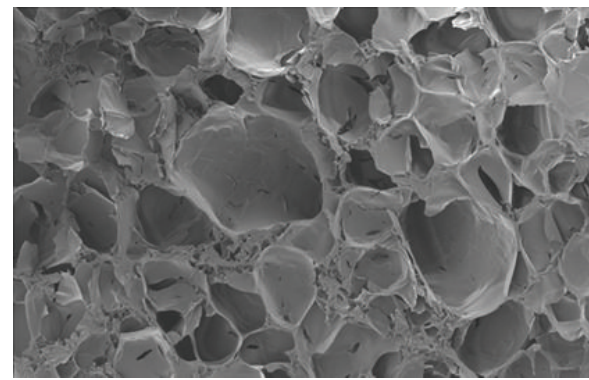

(f)

FIGURE 3: SEM images of surface morphology of chitosan/gelatin sponges (a) SP3, (b) SP2, and (c) SP1. Cross-section morphology of chitosan/gelatin sponges (d) SP3, (e) SP2, and (f) SP1 (scale bar at $100 \mu \mathrm{m}$ ).

TABLE 1: Composition and physical parameter of prepared sponges.

\begin{tabular}{|c|c|c|c|c|}
\hline \multirow{2}{*}{ Sample no. } & \multicolumn{2}{|c|}{ Feeding ratio } & \multirow{2}{*}{$\begin{array}{l}\text { Average water } \\
\text { uptake }(\%)\end{array}$} & \multirow{2}{*}{$\begin{array}{l}\text { Weight } \\
\text { loss (\%) }\end{array}$} \\
\hline & Chitosan & Gelatin & & \\
\hline SP1 & 1 & 3 & 227 & 81.5 \\
\hline SP2 & 1 & 1 & 351 & 66.5 \\
\hline SP3 & 3 & 1 & 2352 & 16 \\
\hline
\end{tabular}

SP1, SP2, and SP3, respectively, in $96 \mathrm{~h}$. The curcumin release increased with increase of gelatin content in prepared sponge (Table 2). This is because the hydrophilicity of curcumin was relatively lower. According to the partition ratio between sponge phase and water phase, the drug in the sponge having a higher gelatin content is preferred to be released, instead of being dissolved by the sponge.

From the results of antibacterial activity studies of prepared sponges against $P$. aeruginosa, it can be seen that the inhibition zone is larger in curcumin composite sponge than that of chitosan sponge without curcumin. It can be also seen that the SP3 sponge exhibited a higher inhibition zone than
TABLE 2: The curcumin release profile of prepared sponges.

\begin{tabular}{lccc}
\hline Time (h) & \multicolumn{3}{c}{ Drug release (\%) } \\
\hline 0 & SP1 & SP2 & SP3 \\
\hline 1 & 0.00 & 0.00 & 0.00 \\
6 & 16.35 & 1.38 & 0.53 \\
24 & 30.85 & 4.47 & 1.62 \\
30 & 43.53 & 10.21 & 7.38 \\
48 & 49.44 & 13.59 & 8.15 \\
54 & 59.44 & 29.73 & 9.21 \\
72 & 63.41 & 33.97 & 14.26 \\
96 & 78.12 & 43.26 & 23.09 \\
\hline
\end{tabular}

those of SP1 and SP2 sponges (Figure 4). The inhibition zones were $21 \mathrm{~mm}$ and $17.5 \mathrm{~mm}$ for curcumin-loaded SP3 and SP3 without curcumin, respectively.

The cytotoxicity of prepared sponges to L929 cells was evaluated using MTT assay method. The results demonstrate the cell viability after $24 \mathrm{~h}$ incubation with medium released 


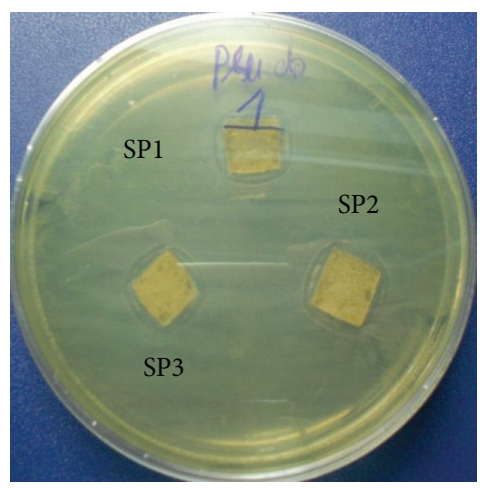

(a)

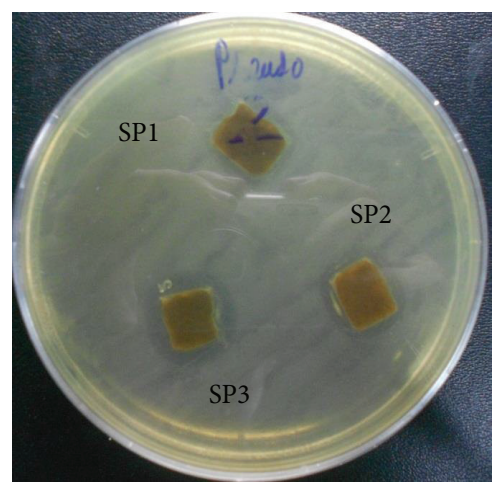

(b)

FIgURE 4: Antibacterial activity of prepared sponges against Pseudomonas aeruginosa: (a) sponges without curcumin and (b) curcuminsponges.

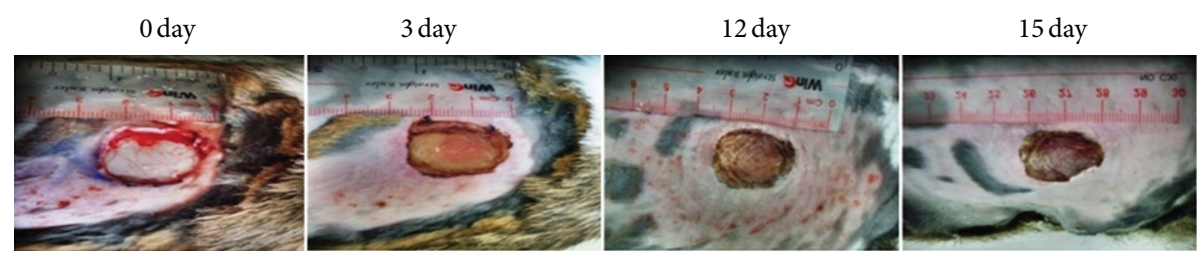

(a)

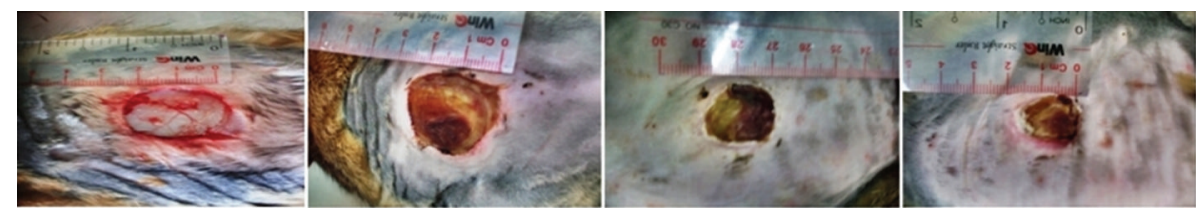

(b)
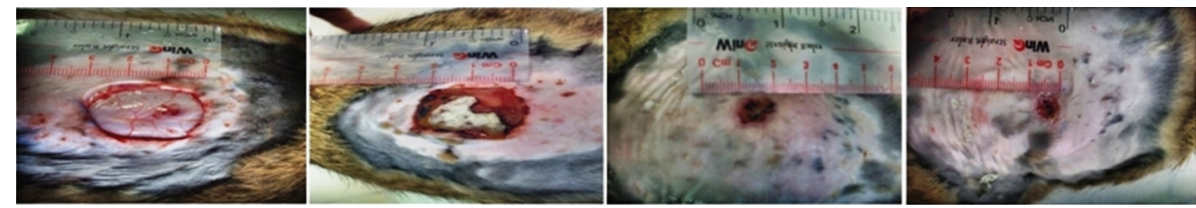

(c)

FIGURE 5: Photographs of macroscopic appearance of wound repair covered with (a) control, (b) blank sponge, and (c) curcumin sponge, at day 0 , day 3 , day 12 , and day 15 , respectively.

from sponges. The results present that cell viability decreased to approximately $90 \%$ in the presence of medium containing sponge without curcumin and curcumin sponge. The cell viability suggests that these sponges have generally low cytotoxicity to the L929 fibroblast cells (data not shown).

The SP3 sponge was selected for wound healing test. The percentage of wound closure in untreated and treated groups was measured on $3 \mathrm{rd}, 12 \mathrm{nd}, 15 \mathrm{th}$, and $21 \mathrm{st}$ post wound day and the results are presented in Table 3 . The wound closure was similar in three groups on 21st post wound day. The wound closure was $99.49 \%, 97.63 \%$, and $94.30 \%$ for curcumin-SP3, blank SP3 and control group, respectively. It was found that the wounds treated with curcumin composite and composite without curcumin have shown faster wound healing than wound untreated group. Moreover, the wound closure on the 12nd and 15th day was significantly increased in curcumin SP3 in comparison with SP3 without curcumin (Figure 5). These results enhance the wound healing activity and could be a good material to be employed as wound dressing.

Histological Examination. The histological examination of three different kinds of wound dressing applied on skin wound of rabbit is presented in Figure 6. As can be seen in Figure 6, the tissue is in the acute stage of wound healing for control (Figure 6(a)). The tissue is under subacute stage of wound healing due to that there are many eosinophil, allergic reactions may be considered (Figure 6(b)). The 


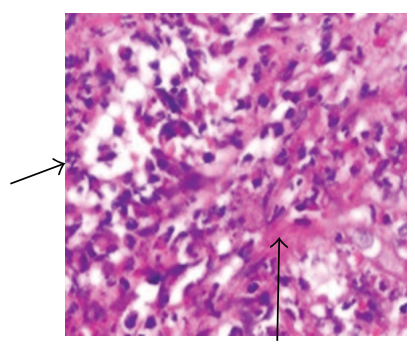

(a)

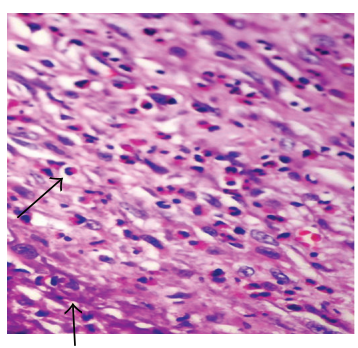

(b)

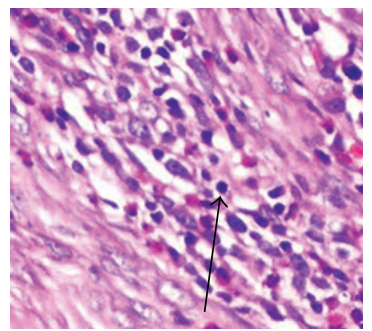

(c)

Figure 6: The H\&E stained sections of twelve-day postsurgery of wound treated with (a) control: Short arrow-polymorphonuclear neutrophil (PMN), long arrow-fibroblast. The tissue is in the acute stage of wound healing. (b) Blank sponge (without curcumin): short arrow-eosinophil, long arrow-necrotic tissue. The tissue is under subacute stage of wound healing due to that there are many eosinophil and allergic reaction may be considered, and (c) curcumin sponge: arrow-lymphocyte, the tissue is under subacute stage of wound healing $(400 x)$

TABLE 3: Wound closure of animal treated with blank sponge and curcumin sponge.

\begin{tabular}{lcccc}
\hline & \multicolumn{4}{c}{ Wound closure (\%) (mean \pm SD) } \\
\hline Time & 3rd day & 12nd day & 15th day & 21st day \\
\hline Control & $15.36 \pm 0.54$ & $30.00 \pm 1.05$ & $48.80 \pm 1.71$ & $94.30 \pm 1.93$ \\
SP3 & $14.79 \pm 0.58$ & $49.41 \pm 1.93$ & $77.96 \pm 3.91$ & $97.63 \pm 4.28$ \\
Curcumin-SP3 & $26.53 \pm 1.14$ & $90.82 \pm 2.11$ & $95.41 \pm 3.62$ & $99.49 \pm 3.81$ \\
\hline
\end{tabular}

wound is under subacute stage for curcumin-loaded sponge (Figure 6(c)). The granulation tissue and collagen alignment were more advanced in the sponge without curcurmin and curcumin-loaded sponge-treated wound as compared to the untreated wounds (control).

\section{Conclusion}

In this work, composite sponge of curcumin/chitosan/gelatin was prepared at the various ratios of chitosan and gelatin. The structure and morphology were characterized using FTIR and SEM. The composite of curcumin, chitosan, and gelatin improved water uptake ability, antibacterial activity, and wound closure. Based on the results of drug release of curcumin, the higher content of gelatin in composite sponge exhibited a faster release behavior up to $240 \mathrm{~min}$. These composite sponges were found to enhance the formation of collagen and wound closure in vivo and therefore improved the wound healing activity.

\section{Acknowledgments}

The authors would like to thank the Nafosted and Industrial University of Ho Chi Minh City for financial support. The authors thank Miss Tu and Miss Minh Nhat for their help on sample preparations.

\section{References}

[1] D. Gopinath, M. R. Ahmed, K. Gomathi, K. Chitra, P. K. Sehgal, and R. Jayakumar, "Dermal wound healing processes with curcumin incorporated collagen films," Biomaterials, vol. 25, no. 10, pp. 1911-1917, 2004.

[2] P. Basnet and N. Skalko-Basnet, "Curcumin: an antiinflammatory molecule from a curry spice on the path to cancer treatment," Molecules, vol. 16, no. 6, pp. 4567-4598, 2011.

[3] A. Kunwar, A. Barik, R. Pandey, and K. I. Priyadarsini, “Transport of liposomal and albumin loaded curcumin to living cells: an absorption and fluorescence spectroscopic study," Biochimica et Biophysica Acta, vol. 1760, no. 10, pp. 1513-1520, 2006.

[4] A. Kumar, S. Dogra, and A. Prakash, "Protective effect of curcumin (Curcuma longa), against aluminium toxicity: possible behavioral and biochemical alterations in rats," Behavioural Brain Research, vol. 205, no. 2, pp. 384-390, 2009.

[5] B. T. Kurien and R. H. Scofield, "Increasing aqueous solubility of curcumin for improving bioavailability," Trends in Pharmacological Sciences, vol. 30, no. 7, pp. 334-335, 2009.

[6] C.-H. Chen, M.-F. Hsieh, Y.-N. Ho et al., "Enhancement of catechin skin permeation via a newly fabricated mPEG-PCL-graft2-hydroxycellulose membrane," Journal of Membrane Science, vol. 371, no. 1-2, pp. 134-140, 2011.

[7] B. Wang, K. Chen, S. Jiang et al., "Chitosan-mediated synthesis of gold nanoparticles on patterned poly(dimethylsiloxane) surfaces," Biomacromolecules, vol. 7, no. 4, pp. 1203-1209, 2006.

[8] H. Yi, L.-Q. Wu, W. E. Bentley et al., "Biofabrication with chitosan," Biomacromolecules, vol. 6, no. 6, pp. 2881-2894, 2005.

[9] T. Dai, M. Tanaka, Y.-Y. Huang, and M. R. Hamblin, "Chitosan preparations for wounds and burns: antimicrobial and woundhealing effects," Expert Review of Anti-Infective Therapy, vol. 9, no. 7, pp. 857-879, 2011.

[10] R. Jayakumar, M. Prabaharan, P. T. Sudheesh Kumar, S. V. Nair, and H. Tamura, "Biomaterials based on chitin and chitosan in 
wound dressing applications," Biotechnology Advances, vol. 29, no. 3, pp. 322-337, 2011.

[11] R. A. A. Muzzarelli, "Chitins and chitosans for the repair of wounded skin, nerve, cartilage and bone," Carbohydrate Polymers, vol. 76, no. 2, pp. 167-182, 2009.

[12] S.-J. Yang, F.-H. Lin, K.-C. Tsai et al., "Folic acid-conjugated chitosan nanoparticles enhanced protoporphyrin IX accumulation in colorectal cancer cells," Bioconjugate Chemistry, vol. 21, no. 4, pp. 679-689, 2010.

[13] Y.-Q. Ye, F.-Y. Chen, Q.-A. Wu et al., "Enhanced cytotoxicity of core modified chitosan based polymeric micelles for doxorubicin delivery," Journal of Pharmaceutical Sciences, vol. 98, no. 2, pp. 704-712, 2009.

[14] H. Zhou, W. Yu, X. Guo et al., "Synthesis and characterization of amphiphilic glycidol-chitosan-deoxycholic acid nanoparticles as a drug carrier for doxorubicin," Biomacromolecules, vol. 11, no. 12, pp. 3480-3486, 2010.

[15] A. Tanaka, T. Nagate, and H. Matsuda, "Acceleration of wound healing by gelatin film dressings with epidermal growth factor," Journal of Veterinary Medical Science, vol. 67, no. 9, pp. 909-913, 2005.

[16] E. J. Chong, T. T. Phan, I. J. Lim et al., "Evaluation of electrospun PCL/gelatin nanofibrous scaffold for wound healing and layered dermal reconstitution," Acta Biomaterialia, vol. 3, no. 3, pp. 321330, 2007.

[17] K. Madhumathi, P. T. Sudheesh Kumar, S. Abhilash et al., "Development of novel chitin/nanosilver composite scaffolds for wound dressing applications," Journal of Materials Science, vol. 21, no. 2, pp. 807-813, 2010.

[18] M. Dai, X. Zheng, X. Xu et al., "Chitosan-alginate sponge: preparation and application in curcumin delivery for dermal wound healing in rat," Journal of Biomedicine and Biotechnology, vol. 2009, Article ID 595126, 8 pages, 2009.

[19] M. C. Silva and C. T. Andrade, "Evaluating conditions for the formation of chitosan/gelatin microparticles," Polimeros, vol. 19, no. 2, pp. 133-137, 2009.

[20] T. V. L. Hima Bindu, M. Vidyavathi, K. Kavitha, T. P. Sastry, and R. V. Suresh kumar, "Preparation and evaluation of ciprofloxacin loaded chitosan-gelatin composite films for wound healing activity," International Journal of Drug Delivery, vol. 2, no. 2, 2011. 

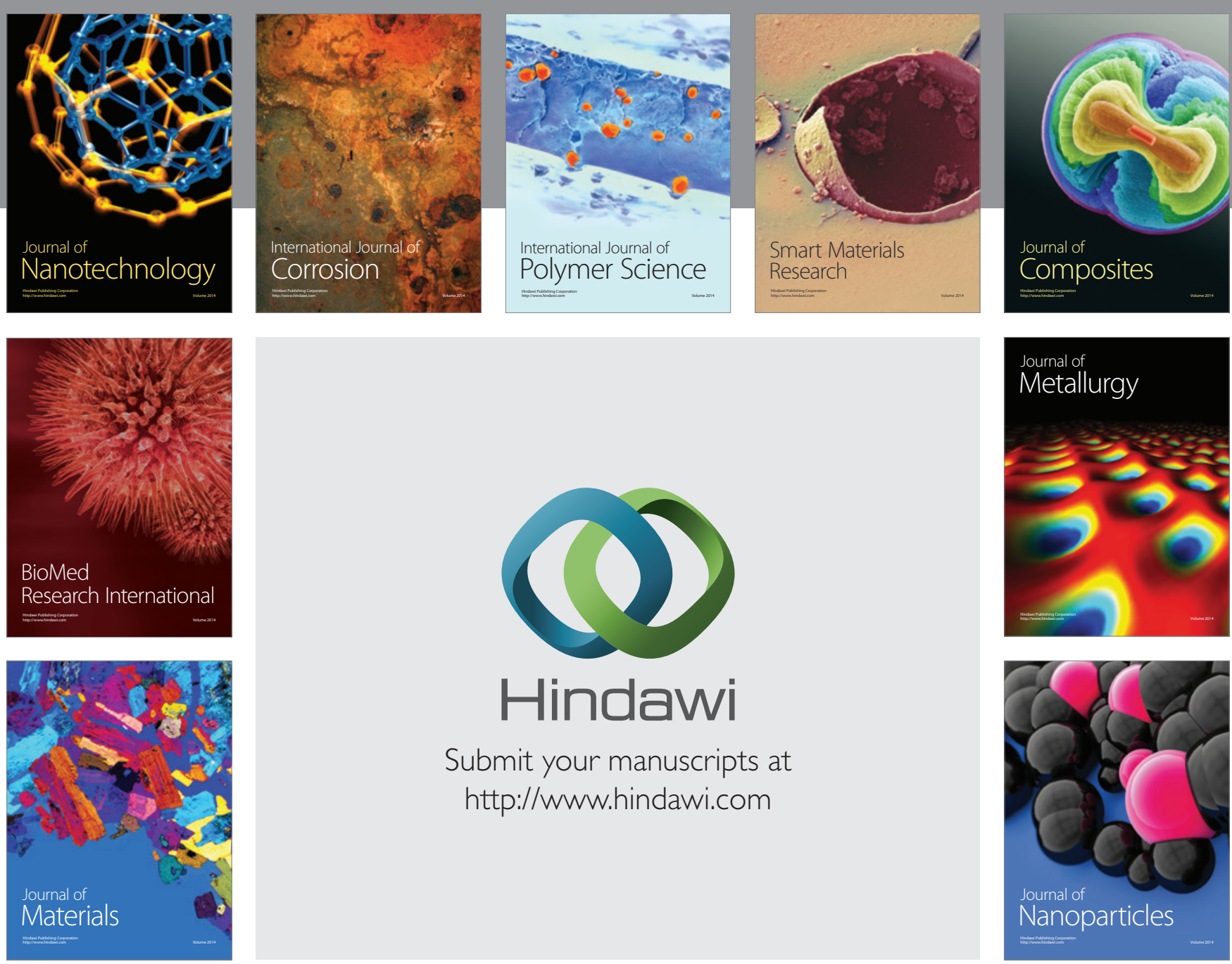

Submit your manuscripts at http://www.hindawi.com
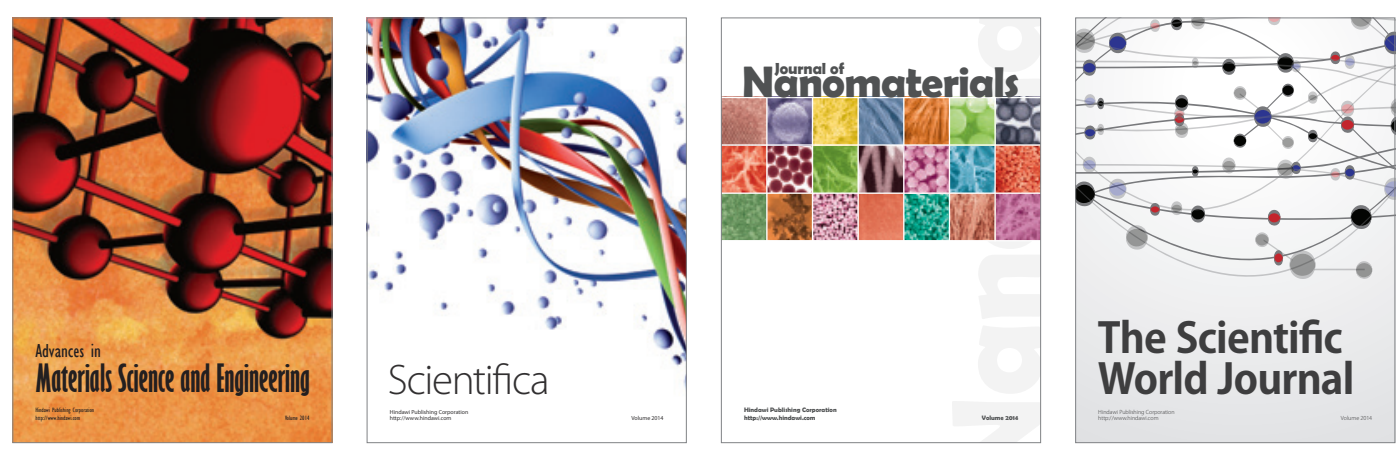

\section{The Scientific World Journal}
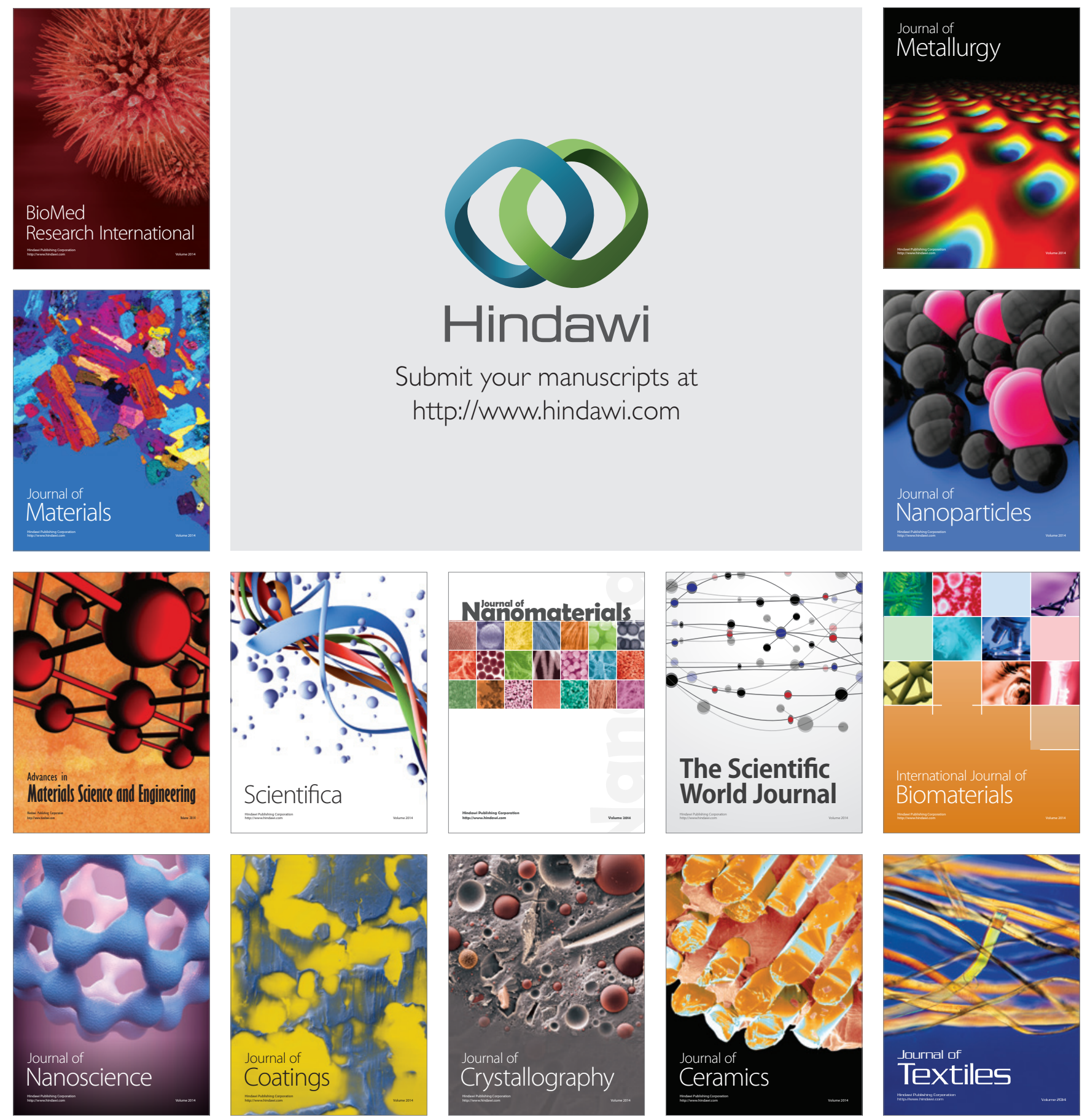